\title{
Correction to: Professor María Teresa Lozano and universal links
}

\author{
Enrique $\operatorname{Artal}^{1}$ (D) Antonio F. $\operatorname{Costa}^{2}$ (D) \\ Milagros Izquierdo ${ }^{3}$ (D)
}

\section{Correction to: RACSAM https://doi.org/10.1007/s13398-017-0446-z}

Unfortunatelly an erratum appears in the foreword of this volume.

We claim that one of the authors of the article On universal groups and three-manifolds, Invent. Math. 87 (1987), no. 3, 441-456 is W. Witten. The correct name of this author is Wilbur Carrington Whitten. W. C. Whitten is not the father of the fields medallist E. Witten, as we claim there.

The online version of the original article can be found under https://doi.org/10.1007/s13398-017-0446-z.

Enrique Artal

artal@unizar.es

Antonio F. Costa

acosta@mat.uned.es

Milagros Izquierdo

milagros.izquierdo@liu.se

1 Departamento de Matemáticas, IUMA, Universidad de Zaragoza, C. Pedro Cerbuna 12, 50009

Zaragoza, Spain

2 Departamento de Matemáticas Fundamentales, UNED, Paseo Senda del Rey 9, 28040 Madrid, Spain

3 Matematiska Institutionen Linköpings Universitet, 58183 Linköpings, Sweden 